\title{
Velocity statistics for point vortices in the local $\alpha$-models of turbulence
}

\author{
G. $\mathrm{CONTI} \dagger *$ and G. BADIN $\dagger$ \\ $\dagger$ Institute of Oceanography, Center for Earth System Research and Sustainability (CEN), University \\ of Hamburg, Hamburg, Germany \\ (16th October 2018)
}

\begin{abstract}
The velocity fluctuations for point vortex models are studied for the $\alpha$-turbulence equations, which are characterized by a fractional Laplacian relation between active scalar and the streamfunction. In particular, we focus on the local dynamics regime. The local dynamics differ from the well-studied case of $2 \mathrm{D}$ turbulence as it allows to consider the true thermodynamic limit, that is, to consider an infinite set of point vortices on an infinite plane keeping the density of the vortices constant. The consequence of this limit is that the results obtained are independent on the number of point vortices in the system. This limit is not defined for $2 \mathrm{D}$ turbulence. We show an analytical form of the probability density distribution of the velocity fluctuations for different degrees of locality. The central region of the distribution is not Gaussian, in contrast to the case of $2 D$ turbulence, but can be approximated with a Gaussian function in the small velocity limit. The tails of the distribution exhibit a power law behavior and self similarity in terms of the density variable. Due to the thermodynamic limit, both the Gaussian approximation for the core and the steepness of the tails are independent on the number of point vortices, but depend on the $\alpha$-model. We also show the connection between the velocity statistics for point vortices uniformly distributed, in the context of the $\alpha$-model in classical turbulence, with the velocity statistics for point vortices non-uniformly distributed. Since the exponent of the power law depends just on $\alpha$, we test the power law approximation obtained with the point vortex approximation, by simulating full turbulent fields for different values of $\alpha$ and we compute the correspondent probability density distribution for the absolute value of the velocity field. These results suggest that the local nature of the turbulent fluctuations in the ocean or in the atmosphere might be deduced from the shape of the tails of the probability density functions.
\end{abstract}

Keywords: Turbulence; $\alpha$-models; Surface quasi-geostrophic dynamics; Vortex dynamics; Velocity Statistics;

\section{Introduction}

Turbulent flows, such as the one observed in astrophysical, geophysical, pipe as well as quantum flows, are characterized by inertial ranges which extend through several scales of motion. In the atmosphere and the ocean, for example, the quasi two-dimensional dynamics has inertial ranges that extend from the large scales, to synoptic and until to submesoscale (e.g. Charney (1971)). Although the development and representation of turbulence is largely unknown, this physical phenomenon drives many important processes such as the transfer of energy to the dissipative scales and the stirring and mixing of tracers. It is then of primary importance trying to understand further the features and the representation of turbulence. In particular, the statistical representation of velocity fluctuations may be used for the formulation of stochastic models representing the dynamics. However, trying to solve analytically these problems is not simple, and simplifications are often required.

Depending on the model used to study turbulence, inverse or forward cascades of the generalized energy and enstrophy can occur in the turbulent inertial ranges. This transfer is manifested with the creation of vorticity dominated structures. For this reason the vorticity dynamics plays a crucial role in the description of turbulence. If we limit our investigation

*Corresponding author. Email: giovanni.conti@uni-hamburg.de 
to $2 \mathrm{D}$ flows, we can introduce an important simplification through the use of point vortices. In this approximation the vorticity field is approximated by a set of localized point vortices, which is the analogous of replacing a continuous mass distribution by a set of localized material points. The resulting dynamics is Hamiltonian and is characterized by the conservation of energy, as well as the linear and angular momentum (Helmholtz 1858). See e.g. Aref $(2007)$, Marchioro and Pulvirenti (2012), Newton (2013) for reviews and Chapman (1978), Badin and Crisciani (2018) for a discussion on symmetries and conservation laws. For analogies with electrodynamics, see e.g. Kraichnan and Montgomery (1980), for applications to astrophysical problems see e.g. Chandrasekhar (1941), Chandrasekhar and von Neumann (1942, 1943), Chavanis (1998), for the statistical dynamics of point vortices in 2D fluid dynamics see e.g. Onsager (1949), Weiss et al. (1998), Chavanis and Sire (2000), Bühler (2002), Esler (2017).

The interactions between point vortices is determined by the model used to study the dynamics. In this work we investigate the velocity field produced by a family of models, the $\alpha$-models. These models are defined using a generalized Euler equation

$$
\frac{\partial \zeta}{\partial t}+J(\psi, \zeta)=0
$$

where $\zeta(x, y, t)$ is an active scalar, $\psi(x, y, t)$ is the streamfunction, and $J(\psi, \zeta)=\partial_{x} \psi \partial_{y} \zeta-$ $\partial_{x} \zeta \partial_{x} \psi$ is the Jacobian determinant. The form of the coupling between these two fields determines the degree of locality of the model. The streamfunction and active scalar fields are related by

$$
\zeta=-(-\Delta \psi)^{\alpha / 2}
$$

where $\boldsymbol{\Delta}$ is the 2D Laplacian operator. In the Fourier space $(2)$ becomes

$$
\hat{\psi}(\boldsymbol{k})=-|\boldsymbol{k}|^{-\alpha} \hat{\zeta}(\boldsymbol{k})
$$

where $\boldsymbol{k}$ is the 2D wavenumber vector, with magnitude $k=|\boldsymbol{k}|$. When $\alpha$ increases, the fields become more decoupled and the problem becomes more spectrally nonlocal. The case $\alpha=2$ represents the widely studied Euler equation, while $\alpha<2$ and $\alpha>2$ represent respectively the local and nonlocal dynamics. For studies of different forms of turbulence emerging from different values of $\alpha$ see, e.g. Pierrehumbert et al. (1994), Smith et al. (2002), Tran and Shepherd (2002), Tran (2004), Tran et al. (2010), Burgess and Shepherd (2013), Burgess et al. (2015), Schorghofer (2000), Venaille et al. (2015), Foussard et al. (2017), Badin and Barry (2018).

The special case of $\alpha=1$ can be used to study the local dynamics of a stratified rapidly rotating flow with zero potential vorticity in the interior domain, and assigned potential temperature at the surface (e.g. the atmospheric tropopause or the oceanic surface). In this case the scalar field $\zeta$ represents the potential temperature. This model is called Surface Quasi Geostrophic (SQG) model (Blumen 1978, Held et al. 1995, Lapeyre 2017, Badin and Crisciani 2018). The analysis of the kinetic energy spectra for this model shows a characteristic forward energy cascade (Tulloch and Smith 2006, Capet et al. 2008), with consequent formation of small structures in the flow. This behaviour is complementary to the one of the $2 \mathrm{D}$ turbulence, and makes the SQG model a possible candidate for the forward cascade of temperature variance and the formation of frontal structures. SQG is also a candidate for the explanation of the submesoscale dynamics (where the term submesoscale is here used in its oceanographic meaning, see e.g. McWilliams $(2016)$ ), which is also important for the mixing of passive tracers (Badin et al. 2011, Shcherbina et al. 2015 , Mukiibi et al. 2016$)$. For a study on the relationship between quasi geostrophic (QG) and SQG turbulence, see e.g. Badin (2014). For the Hamiltonian and Nambu structure of SQG, see e.g. Blender and Badin (2015). The stability of SQG vortices was studied by e.g. Carton (2009), Dritschel (2011), Harvey and Ambaum (2011), Harvey et al. (2011), Bembenek et al. (2015), Carton et al. (2016), Badin and Poulin 
(2018). SQG point vortices have been studied by e.g. Lim and Majda (2001), Taylor and Llewellyn Smith (2016), Badin and Barry (2018).

Mathematically, SQG shows interesting analogies with the 3D Euler equation (Constantin et al. 1994a). This analogy sparked a large interest, as it suggests that the study of the regularity of the SQG model could provide hints for the formation of singularities in the 3D Euler equation, see e.g. Constantin et al. (1994a b), Pierrehumbert et al. (1994), Majda and Tabak (1996), Ohkitani and Yamada (1997), Constantin et al. (1998), Constantin and Wu (1999), Córdoba and Fefferman (2002a b), Córdoba and Córdoba (2004), Rodrigo and Fefferman (2004), Córdoba et al. (2005), Rodrigo (2005), $\mathrm{Wu}(2005)$, Deng et al. $(2006)$, Dong and Li (2008), Ju (2006), Li (2009), Marchand (2008a b), Scott (2011), Constantin et al. (2012), Ohkitani (2012), Scott and Dritschel (2014), Badin and Barry (2018).

The aim of this work is the investigation of velocity statistics for turbulent flow, with the simplification of point vortices randomly distributed and an interaction between vortices ruled by a family of $\alpha$-model. One important difference that emerges from the computation is that, away from $\alpha=2$, it is really possible to consider a proper thermodynamics limit, that is, we can consider an infinite set of point vortices on an infinite plane keeping the density of the vortices constant. As a consequence of this limit, all the results we obtain are independent on the number of point vortices in the domain considered. Given their physical interest, we will focus on local dynamics, with particular attention to the SQG model. Throughout the article we will follow closely the approach used by Chavanis and Sire (2000), Skaugen and Angheluta (2016a) to study 2D turbulence using a random distribution of point vortices, and the one of Chavanis (2009) to study the statistics of the gravitational force induced by inhomogeneous distribution of sources in $d$ dimensions. To test the reliability of the point vortex approximation, we have also simulated (1) and (2) for three different values of $\alpha$ computing then the corresponding probability density functions (PDFs) for the absolute value of the velocity fields to compare with their analytical approximation.

\section{Statistical Formulation}

\subsection{Formal solution}

Consider a point vortex with circulation $\gamma$ placed at $\boldsymbol{r}_{0}$, so that

$$
\zeta(\boldsymbol{r})=\gamma \delta\left(\boldsymbol{r}-\boldsymbol{r}_{0}\right)
$$

where $\delta$ is the Dirac delta function and $\zeta$ the active scalar field at the position $\boldsymbol{r}$. Even if $\zeta$ can represent active scalars which might have a different physical meaning than vorticity, throughout the article we will still call the objects arising from relation (4) as point "vortices".

For the $\alpha$-models, the Green's functions $G^{(\alpha)}(\boldsymbol{r})$ are introduced following Iwayama and Watanabe (2010) as

$$
\begin{aligned}
G^{(2)}\left(\boldsymbol{r}, \boldsymbol{r}^{\prime}\right) & =\frac{1}{2 \pi} \ln \left(\boldsymbol{r}-\boldsymbol{r}^{\prime}\right)+C, \\
G^{(\alpha)}\left(\boldsymbol{r}, \boldsymbol{r}^{\prime}\right) & =\Psi(\alpha)\left(\boldsymbol{r}-\boldsymbol{r}^{\prime}\right)^{\alpha-2}, \quad \alpha \neq 2,
\end{aligned}
$$

with $C$ arbitrary constant and

$$
\Psi(\alpha)=-\left\{2^{\alpha}\left[\Gamma\left(\frac{\alpha}{2}\right)\right]^{2} \sin \left(\frac{\alpha \pi}{2}\right)\right\}^{-1},
$$

so that the streamfunction of the flow can be expressed as

$$
\psi(\boldsymbol{r})=\int G^{(\alpha)}\left(\boldsymbol{r}, \boldsymbol{r}^{\prime}\right) \zeta\left(\boldsymbol{r}^{\prime}\right) d \boldsymbol{r}^{\prime}
$$






Figure 1. $\Psi(\alpha)$, as defined by 7 , for different $\alpha$-models with $\alpha \in(0,2)$.

Figure 1 shows the behaviour of $\Psi(\alpha)$, for different values of $\alpha$. In the considered interval, $\Psi(\alpha)$ results to be always negative. Notice that when $\alpha \rightarrow 2$ then $\Psi(\alpha) \rightarrow-\infty$, there is a discontinuity in the Green's functions between the local, $\alpha<2$, and nonlocal, $\alpha>2$, dynamics. Using the stream function we can write the velocity field generated in a given location by the point vortex following Badin and Barry (2018) as

$$
\begin{aligned}
\phi^{(2)} & =-\frac{\gamma}{2 \pi} \frac{\boldsymbol{r}_{\perp}}{r^{2}} \\
\phi^{(\alpha)} & =-\gamma \Psi(\alpha) \frac{\boldsymbol{r}_{\perp}}{r^{(4-\alpha)}}, \quad \alpha \neq 2 .
\end{aligned}
$$

In (10), the $\perp$ subscript denotes the clockwise rotation of a vector by $\pi / 2$.

In the following we will consider an ensemble of $N$ identical point vortices, and following Chavanis and Sire (2000), Skaugen and Angheluta (2016a) we shall assume:

(i) a "neutral" system, i.e. a system in which its vortices can exhibit only two values for the circulation, $+|\gamma|$ and $-|\gamma|$, in equal proportion to avoid solid rotation;

(ii) vortices randomly distributed with uniform probability on a disk of radius $R$;

(iii) uncorrelated vortices, that is, the positions of the vortices inside the domain are uncorrelated and the probability of the N-point configurational distribution can be seen as a product of the probability $p(\boldsymbol{r})$ of finding a vortex.

Assumptions (i) and (ii) state that a vortex with circulation $-|\gamma|$ located in $\boldsymbol{r}$ produces the same velocity field of a vortex with circulation $+|\gamma|$ located in $-\boldsymbol{r}$, and since the two species of vortices are randomly distributed with uniform probability we have the statistical equivalence of the two groups of vortices, and we can proceed further considering just a single species of vortices. Assumption (iii) is used in defining the probability density for the velocity, as we will show in a moment.

We shall also assume:

(iv) that, without loss of generality, the velocity $\boldsymbol{V}$ is computed at the center of the domain.

From (ii) follows that the probability density of having a vortex at position $\boldsymbol{r}$ is

$$
p(\boldsymbol{r})=\frac{1}{\pi R^{2}},
$$

and the vortices density is

$$
n=\frac{N}{\pi R^{2}} .
$$

The velocity field $\boldsymbol{V}$ produced by the $N$ vortices at a given point is the sum of the velocity 
fields at that point

$$
\boldsymbol{V}=\sum_{i=1}^{N} \boldsymbol{\phi}_{i}^{(\alpha)}, \quad \alpha \in(0,2] .
$$

Under these considerations the $\operatorname{PDF} P_{N}^{(\alpha)}(\boldsymbol{V})$, that gives the probability $P_{N}^{(\alpha)}(\boldsymbol{V}) d \boldsymbol{V}$ for the velocity $\boldsymbol{V}$ to fall between $\boldsymbol{V}$ and $\boldsymbol{V}+d \boldsymbol{V}$, can be written as

$$
P_{N}^{(\alpha)}(\boldsymbol{V})=\int \prod_{i=1}^{N} p\left(\boldsymbol{r}_{i}\right) \delta\left(\boldsymbol{V}-\sum_{i=1}^{N} \boldsymbol{\phi}_{i}^{(\alpha)}\right) d \boldsymbol{r},
$$

where the assumption (iii) has been used to factorized the PDF for the N-point configuration. To simplify (14), decoupling the integral, we can use the integral form of the Dirac delta function

$$
\delta(\boldsymbol{x})=\frac{1}{(2 \pi)^{2}} \int e^{-i \boldsymbol{\rho} \cdot \boldsymbol{x}} d \boldsymbol{\rho} .
$$

Inserting this expression in (14) yields

$$
P_{N}^{(\alpha)}(\boldsymbol{V})=\frac{1}{4 \pi^{2}} \int A_{N}^{(\alpha)}(\boldsymbol{\rho}) e^{-i \boldsymbol{\rho} \cdot \boldsymbol{V}} d \boldsymbol{\rho}
$$

with

$$
\begin{aligned}
A_{N}^{(\alpha)}(\boldsymbol{\rho}) & =\left(\int_{|\boldsymbol{r}|=0}^{R} e^{i \boldsymbol{\rho} \cdot \phi^{(\alpha)}} p(\boldsymbol{r}) d \boldsymbol{r}\right)^{N} \\
& =\left(\frac{1}{\pi R^{2}} \int_{|\boldsymbol{r}|=0}^{R} e^{i \boldsymbol{\rho} \cdot \phi^{(\alpha)}} d \boldsymbol{r}\right)^{N}
\end{aligned}
$$

where we have made use of (11). Since the probability density for the vortices position is normalized to the unity, (17) can be written as

$$
\begin{aligned}
A_{N}^{(\alpha)}(\boldsymbol{\rho}) & =\left(1-\frac{1}{\pi R^{2}} \int_{|\boldsymbol{r}|=0}^{R}\left(1-e^{i \boldsymbol{\rho} \cdot \phi^{(\alpha)}}\right) d \boldsymbol{r}\right)^{N} \\
& =\left(1-\frac{n}{N} \int_{|\boldsymbol{r}|=0}^{R}\left(1-e^{i \boldsymbol{\rho} \cdot \phi^{(\alpha)}}\right) d \boldsymbol{r}\right)^{N} .
\end{aligned}
$$

When the thermodynamic limit is considered, that is

$$
N \rightarrow \infty, \quad R \rightarrow \infty, \quad \text { with } n=\text { const }
$$

from the special limit in 18 we obtain

$$
A^{(\alpha)}(\boldsymbol{\rho})=e^{-n C^{(\alpha)}(\boldsymbol{\rho})},
$$

with

$$
C^{(\alpha)}(\boldsymbol{\rho})=\int_{|\boldsymbol{r}|=0}^{\infty}\left(1-e^{i \boldsymbol{\rho} \cdot \boldsymbol{\phi}^{(\alpha)}}\right) d \boldsymbol{r},
$$

where we have dropped the subscript $\mathrm{N}$ to indicate that the thermodynamics limit was been taken.

Notice that for $\alpha=2$ the thermodynamic limit $(19)$ does not exist due to logarithmic divergence with the number of vortices (Jimnez 1996, Min et al. 1996, Weiss et al. 1998). Therefore, in the particular case of 2D turbulence, (20) must not be regarded as a true limit. 
For the same reason, for $\alpha=2$ it is not possible to consider the upper limit in the integral (21) as equal to infinity.

In order to proceed further in the computation of $P^{(\alpha)}(\boldsymbol{V})$, we have to evaluate $C^{(\alpha)}$. As suggested by Chavanis and Sire (2000), Chavanis (2009) it is convenient to carry on the computation changing the variable of integration from $\boldsymbol{r}$ to $\phi$. From $(9)$ and $(10)$ it follows that the Jacobi determinant of the transformation is

$$
\begin{aligned}
& \left\|\frac{\partial(\boldsymbol{r})}{\partial\left(\phi^{(2)}\right)}\right\|=-\frac{\gamma^{2}}{4 \pi^{2}}\left(\phi^{(2)}\right)^{-4}, \\
& \left\|\frac{\partial(\boldsymbol{r})}{\partial\left(\boldsymbol{\phi}^{(\alpha)}\right)}\right\|=-\frac{(\gamma|\Psi(\alpha)|)^{\frac{2}{3-\alpha}}}{3-\alpha}\left(\phi^{(\alpha)}\right)^{-2\left(\frac{4-\alpha}{3-\alpha}\right)}, \quad \alpha \neq 2,
\end{aligned}
$$

and the integrals $(21)$ become

$$
C^{(2)}(\boldsymbol{\rho})=\frac{\gamma^{2}}{4 \pi^{2}} \int_{\left|\phi^{(2)}\right|=\frac{\gamma}{2 \pi R}}^{\infty}\left(1-e^{i \boldsymbol{\rho} \cdot \boldsymbol{\phi}^{(2)}}\right)\left(\phi^{(2)}\right)^{-4} d \boldsymbol{\phi}^{(2)},
$$

and

$$
C^{(\alpha)}(\boldsymbol{\rho})=\frac{(\gamma|\Psi(\alpha)|)^{\frac{2}{3-\alpha}}}{3-\alpha} \int_{\left|\phi^{(\alpha)}\right|=0}^{\infty}\left(1-e^{i \boldsymbol{\rho} \cdot \boldsymbol{\phi}^{(\alpha)}}\right)\left(\phi^{(\alpha)}\right)^{-2\left(\frac{4-\alpha}{3-\alpha}\right)} d \boldsymbol{\phi}^{(\alpha)}, \alpha \neq 2 .
$$

We first consider (24). Switching to polar coordinates, choosing the radial coordinate in the direction of $\boldsymbol{\rho}$, and defining with $\theta$ the angle between $\boldsymbol{\rho}$ and $\boldsymbol{\phi}^{(2)}$, we obtain

$$
\begin{aligned}
C^{(2)}(\boldsymbol{\rho}) & =\frac{\gamma^{2}}{(2 \pi)^{2}} \int_{\frac{\gamma}{2 \pi R}}^{\infty} \int_{0}^{2 \pi}\left(1-e^{i \rho \phi \cos \theta}\right)\left(\phi^{(2)}\right)^{-3} d \theta d \phi^{(2)} \\
& =\frac{\gamma^{2}}{2 \pi} \int_{\frac{\gamma}{2 \pi R}}^{\infty}\left(1-J_{0}\left(\rho \phi^{(2)}\right)\right)\left(\phi^{(2)}\right)^{-3} d \phi^{(2)},
\end{aligned}
$$

where $J_{0}$ is the Bessel function of the first kind and order zero. Finally, substituting $x=\rho \phi^{(2)}$,

$$
\begin{aligned}
C^{(2)}(\boldsymbol{\rho}) & =2 \pi\left(\frac{\gamma \rho}{2 \pi}\right)^{2} \int_{\frac{\gamma}{2 \pi R}}^{\infty}\left(1-J_{0}(x)\right) x^{-3} d x \\
& =2 \pi\left(\frac{\gamma \rho}{2 \pi}\right)^{2} \kappa^{(2)}(R),
\end{aligned}
$$

where $\kappa^{(2)}(R)$ is a dimensionless number given by the integral in (27). Using a similar strategy for the case $\alpha \neq 2$, we switch to polar coordinate and extend the limits of the integration in (25) to get

$$
\begin{aligned}
C^{(\alpha)}(\boldsymbol{\rho}) & =\frac{(\gamma|\Psi(\alpha)|)^{\frac{2}{3-\alpha}}}{3-\alpha} \int_{0}^{\infty} \int_{0}^{2 \pi}\left(1-e^{i \rho \phi \cos \theta}\right)\left(\phi^{(\alpha)}\right)^{-\left(\frac{5-\alpha}{3-\alpha}\right)} d \theta d \phi^{(\alpha)} \\
& =2 \pi \frac{(\gamma|\Psi(\alpha)|)^{\frac{2}{3-\alpha}}}{3-\alpha} \int_{0}^{\infty}\left(1-J_{0}\left(\rho \phi^{(\alpha)}\right)\right)\left(\phi^{(\alpha)}\right)^{-\left(\frac{5-\alpha}{3-\alpha}\right)} d \phi^{(\alpha)}, \alpha \neq 2 .
\end{aligned}
$$

Substituting $x=\rho \phi^{(\alpha)}$ yields

$$
\begin{aligned}
C^{(\alpha)}(\boldsymbol{\rho}) & =2 \pi A^{(\alpha)} \rho^{\frac{2}{3-\alpha}} \int_{0}^{\infty}\left(1-J_{0}(x)\right) x^{-\left(\frac{5-\alpha}{3-\alpha}\right)} d x \\
& =2 \pi A^{(\alpha)} \kappa^{(\alpha)} \rho^{\frac{2}{3-\alpha}}, \quad \alpha \neq 2,
\end{aligned}
$$




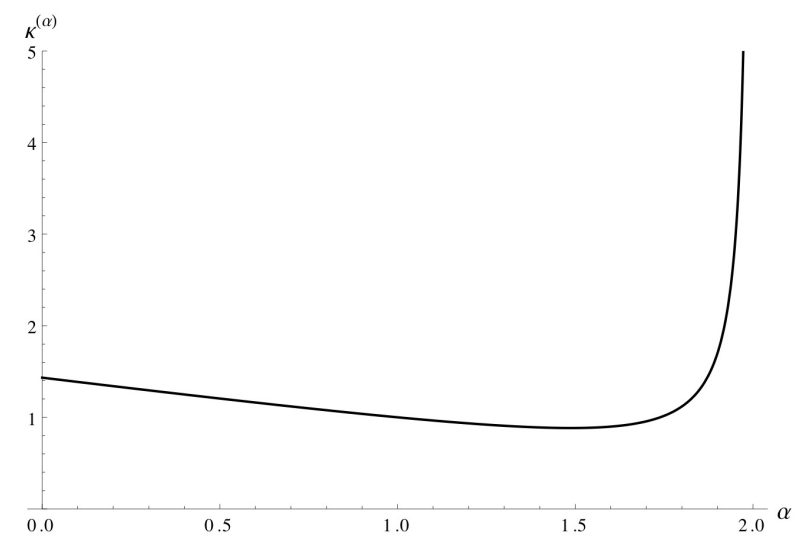

Figure 2. $\kappa^{(\alpha)}$, as defined by 32 , for different $\alpha$-models with $\alpha \in(0,2)$.

where

$$
A^{(\alpha)}=\frac{(\gamma|\Psi(\alpha)|)^{\frac{2}{3-\alpha}}}{3-\alpha}
$$

and $\kappa^{(\alpha)}$ is a dimensionless number given by the integral in $(29)$, that depends parametrically on $\alpha$.

The formal solution for the PDF can thus be written as

$$
\begin{aligned}
P^{(\alpha)}(\boldsymbol{V}) & =\frac{1}{(2 \pi)^{2}} \int A^{(\alpha)}(\boldsymbol{\rho}) e^{i \boldsymbol{\rho} \cdot \boldsymbol{V}} d \boldsymbol{\rho} \\
& =\frac{1}{(2 \pi)^{2}} \int_{0}^{2 \pi} \int_{0}^{\infty} \rho e^{-n C^{(\alpha)}(\boldsymbol{\rho})} e^{i \rho V \cos \theta} d \rho d \theta,
\end{aligned}
$$

with $\alpha \in(0,2]$, and $C^{(\alpha)}$ given by either (27) or $(29)$, and the introduction of polar coordinates. From now on we concentrate on the interval $\alpha \in(0,2)$ if not explicitly specified differently.

The function $\kappa^{(\alpha)}$ that appears in $(29)$ can be evaluated as (see, e.g. Skaugen and Angheluta (2016a))

$$
\begin{aligned}
& \kappa^{(\alpha)}=\int_{0}^{\infty}\left(1-J_{0}(x)\right) x^{-\left(\frac{5-\alpha}{3-\alpha}\right)} d x \\
& =-\frac{1}{\pi} \sin \left[\frac{\pi}{2}\left(\frac{1-\alpha}{3-\alpha}\right)\right] \Gamma\left(\frac{-2}{3-\alpha}\right) B\left[\frac{1}{2}\left(\frac{5-\alpha}{3-\alpha}\right), \frac{1}{2}\right],
\end{aligned}
$$

where $B$ is the Beta function.

Figure 2 shows the behaviour of $\kappa^{(\alpha)}$ for different $\alpha$-models with $\alpha \in(0,2)$. The function $\kappa^{(\alpha)}$ shows positive values in all the interval, decreasing for increasing values of $\alpha$ until $\alpha \approx$ 1.48, where it exhibits a minimum, and then increasing for the rest of the interval. The function diverges for $\alpha \rightarrow 2$.

Because $\kappa^{(\alpha)}>0$ in all the $(0,2)$ interval, for these values of $\alpha$ we can solve for the tails of the distribution following the same procedure used by Chavanis and Sire (2000), Skaugen and Angheluta (2016a). This will be done in the next Section.

\subsection{Tails of the distribution}

In order to study the tails of the distribution, we start by considering the following physical argument. High velocities in the tails are induced only by nearby vortices. As the probability to find nearby vortices to a given vortex is low, in first approximation only one neighbouring 
vortex will be important. The probability that the nearest neighbour occur between $r$ and $r+d r$ is $p^{(\alpha)}(r) d r$, where

$$
p^{(\alpha)}(r)=2 \pi n r e^{-\pi n r^{2}} .
$$

In (33), the superscript $\alpha$ indicates the implicit dependence on the degree of locality of the model in the inversion between the distance between vortices $r$ and the velocity $V$.

When $r$ is small, the nearest neighbour distribution becomes

$$
p_{0}^{(\alpha)}(r) \sim 2 \pi n r .
$$

The subscript 0 indicates that (34) is the leading term in the expansion for small $r$ of the full nearest neighbour distribution. With these premises we can write thus for the tails

$$
P^{(\alpha)}(|\mathbf{V}|=V) \sim p_{0}^{(\alpha)}(r(V))\left|\frac{d r(V)}{d V}\right|
$$

that is

$$
P^{(\alpha)}(|\boldsymbol{V}|=V) \sim \frac{2 \pi n(\gamma|\Psi(\alpha)|)^{\frac{2}{3-\alpha}}}{3-\alpha} V^{-\frac{5-\alpha}{3-\alpha}} .
$$

From (36) it is visible that the absolute value of the exponent of the power law tail increases thus with $\alpha$.

The PDF for the absolute value of the velocity for a fixed value of $|\boldsymbol{V}|=V$ is related to the PDF of the velocity by $P^{(\alpha)}(|\boldsymbol{V}|=V)=2 \pi V P^{(\alpha)}(\boldsymbol{V})$, so that

$$
P^{(\alpha)}(\mathbf{V}) \sim \frac{n(\gamma|\Psi(\alpha)|)^{\frac{2}{3-\alpha}}}{3-\alpha} V^{-2 \frac{4-\alpha}{3-\alpha}} .
$$

The distribution (34) can be recovered from (33) not only when $r$ is small, but also when $\pi n r^{2}$ is small. This implies that approximation (34) is valid also when the distance of the nearest neighbour is not small but the vortices are diluted in the domain. The magnitude of the velocity for which (37) start to fail can be calculated expanding the exponential in (33) to the first order and determining when the first order approximation has half of the weight of the leading order term. Then

$$
p^{(\alpha)}(r) \sim p_{0}^{(\alpha)}(r)+p_{1}^{(\alpha)}(r)=2 \pi n r-2 \pi^{2} n^{2} r^{3},
$$

and, using (35),

$$
P^{(\alpha)}(|\boldsymbol{V}|=V) \sim P_{0}^{(\alpha)}(|\boldsymbol{V}|=V)-P_{1}^{(\alpha)}(|\boldsymbol{V}|=V),
$$

where $P_{0}^{(\alpha)}$ is the right hand side of 36 and

$$
P_{1}^{(\alpha)}(|\boldsymbol{V}|=V)=\frac{2 \pi^{2} n^{2}(\gamma|\Psi(\alpha)|)^{\frac{4}{3-\alpha}}}{3-\alpha} V^{-\frac{7-\alpha}{3-\alpha}} .
$$

Using the condition

$$
P_{1}^{(\alpha)}\left(|\boldsymbol{V}|=V_{\text {cut }}\right)=\frac{1}{2} P_{0}^{(\alpha)}\left(|\boldsymbol{V}|=V_{\text {cut }}\right),
$$

one obtains

$$
V_{c u t}=(2 n \pi)^{\frac{3-\alpha}{2}} \gamma|\Psi(\alpha)| .
$$

For strongly local dynamics one observes the formation of clusters of vortices (see e.g. the numerical simulations shown in Section 33, so that the physical argument used at the beginning of this Section, which is based on the presence of a single nearest neighbour, does not hold any longer. Even in this case, it is however still possible to obtain analytical results which are 
independent on the assumption of a single nearest neighbour. These results can be obtained from the explicit evaluation of some integrals for the specific case $V \gg 1$. It is shown in the Appendix that in this asymptotic regime one recovers the shape for the tails of the PDF given by $(37)$.

\subsubsection{Self-similarity of the tails}

The results so far obtained show an interesting self-similarity of the tails of the PDF with respect to the density $n$. If we rescale the velocity using the density

$$
\tilde{\boldsymbol{V}}=\boldsymbol{V} / n^{\frac{3-\alpha}{5-\alpha}}
$$

the PDF of the velocity can be rewritten as

$$
P^{(\alpha)}(\boldsymbol{V}) \sim n^{-\frac{3-\alpha}{5-\alpha}} \mathcal{P}\left(\boldsymbol{V} / n^{\frac{3-\alpha}{5-\alpha}}\right),
$$

which shows that the PDF is self-similar with respect to the density $n$. The rescaled distribution is

$$
\mathcal{P}(\tilde{\boldsymbol{V}}) \sim V^{-2 \frac{4-\alpha}{3-\alpha}}
$$

which is independent of $n$.

Similarly, for the probability density of the module of the velocity we can set

$$
\tilde{\boldsymbol{V}}=\boldsymbol{V} / n^{\frac{3-\alpha}{2}}
$$

so that

$$
P^{(\alpha)}(|\boldsymbol{V}|=V) \sim n^{-\frac{3-\alpha}{2}} \mathcal{P}\left(\boldsymbol{V} / n^{\frac{3-\alpha}{2}}\right)
$$

and

$$
\mathcal{P}(\tilde{\boldsymbol{V}}) \sim \tilde{V}^{-\frac{5-\alpha}{3-\alpha}}
$$

which is independent of $n$.

This self-similarity is particularly meaningful for the $\alpha$-models thanks to the validity of the thermodynamics limit, following which the results do not depend on the number of vortices but on their density $n$.

\subsection{Core of the distribution and small velocity limit}

In order to explore the core of the distribution we can integrate the angular part of (31) to obtain

$$
P^{(\alpha)}(\boldsymbol{V})=\frac{1}{2 \pi} \int_{0}^{\infty} \exp \left(-c^{(\alpha)} \rho^{\frac{2}{3-\alpha}}\right) \rho J_{0}(\rho V) d \rho
$$

where we have set

$$
c^{(\alpha)}=n 2 \pi \kappa^{(\alpha)} \frac{(\gamma|\Psi(\alpha)|)^{\frac{2}{3-\alpha}}}{3-\alpha},
$$

and with $\kappa^{(\alpha)}$ defined in 32 . Since the core of the distribution describes the behaviour for small velocity, we can expand the Bessel function for small argument $\rho V \ll 1$ to get

$$
P^{(\alpha)}(\boldsymbol{V}) \sim \frac{1}{2 \pi} \int_{0}^{\infty} \exp \left(-c^{(\alpha)} \rho^{\frac{2}{3-\alpha}}\right) \rho\left(1-\frac{(\rho V)^{2}}{4}+\frac{(\rho V)^{4}}{64}\right) d \rho
$$


The evaluation of the integral above yields the PDF

$$
P^{(\alpha)}(\boldsymbol{V}) \sim \frac{\left(c^{(\alpha)}\right)^{\alpha-3} \Gamma(4-\alpha)}{4 \pi}\left(1-\frac{\left(c^{(\alpha)}\right)^{\alpha-3}}{8} \frac{\Gamma(7-2 \alpha)}{\Gamma(4-\alpha)} V^{2}+\frac{\left(c^{(\alpha)}\right)^{2(\alpha-3)}}{192} \frac{\Gamma(10-3 \alpha)}{\Gamma(4-\alpha)} V^{4}\right) .
$$

The form of the PDF (52) differs from a Gaussian Taylor series already by the third term in the expansion, implying that the core of the distribution is not Gaussian, differing then from the case $\alpha=2$.

However, in the small velocity limit the distribution can be approximated to a Gaussian function considering only the first two terms in the expansion (52) that is

$$
P^{(\alpha)}(\boldsymbol{V}) \sim \frac{\left(c^{(\alpha)}\right)^{\alpha-3} \Gamma(4-\alpha)}{4 \pi} \exp \left(-\frac{1}{4\left(c^{(\alpha)}\right)^{3-\alpha}} \frac{\Gamma(6-2 \alpha)}{\Gamma(3-\alpha)} V^{2}\right) .
$$

Notice that when $\alpha \rightarrow 0$ the core of the distribution becomes more peaked to highlight that the dynamics is more local and the velocity field between the vortices is characterized mostly by lower velocity.

Similarly to the analysis for the tails of the distribution, we can ask for which value of $V$ the Gaussian function is no longer a valid approximation. Equating the quartic term in (52) with half of the quadratic term yields

$$
V_{\text {gcut }}=2 \sqrt{3}\left(c^{(\alpha)}\right)^{\frac{3-\alpha}{2}} \sqrt{\frac{\Gamma(7-2 \alpha)}{\Gamma(10-3 \alpha)}} .
$$

As shown in the next Section, $V_{\text {gcut }}$ increases with $\alpha$. For $\alpha \rightarrow 2$ the value of $V_{\text {gcut }}$ instead diverges. This divergence is the consequence of the divergence of the Gaussian core of the distribution for $2 D$ turbulence in the thermodynamic limit.

\subsection{Comparison between full and approximated distributions}

In the previous paragraphs it was found that in the thermodynamics limit, the distribution for the velocity can be approximated in the small velocity limit and for the tail by

$$
P^{(\alpha)}(\boldsymbol{V}) \sim \begin{cases}\frac{\left(c^{(\alpha)}\right)^{\alpha-3} \Gamma(4-\alpha)}{4 \pi} \exp \left(-\frac{1}{4\left(c^{(\alpha)}\right)^{3-\alpha}} \frac{\Gamma(6-2 \alpha)}{\Gamma(3-\alpha)} V^{2}\right), & V<V_{\text {gcut }} \\ \frac{n(\gamma|\Psi(\alpha)|)^{\frac{2}{3-\alpha}}}{3-\alpha} V^{-2 \frac{4-\alpha}{3-\alpha}}, & V>V_{\text {cut }},\end{cases}
$$

for $\alpha \in(0,2), c^{(\alpha)}$ defined as in (50), $\Psi(\alpha)$ defined as in (7), $V_{\text {gcut }}$ and $V_{\text {cut }}$ as in (54) and (42) respectively. Both the variance and the slope of the tail depend thus on the degree of locality $\alpha$.

Figure 3 shows a comparison between the full distribution obtained upon the integration of (49) and approximation (55).

Figure 3 a shows the full PDFs (full lines) and the PDFs obtained from the small velocity limit approximation (dashed lines) for $\alpha=0.5,1,1.5$. For fixed values of the density $n$ and circulation $\gamma$, the distributions tends to be broader for increasing values of $\alpha$. This is better seen in Figure 3b, which shows the distributions in logarithmic scale in order to highlight the behaviour of the tails and their power law approximations. For increasing values of $\alpha$, the tails tend to become less important. This can be better understood by considering the ratio between the limits of validity of the two approximations $V_{c u t}$ and $V_{\text {gcut }}$, which is shown Figure 3k. It should be noted that this ratio is independent on the values of the density and circulation. When $\alpha \rightarrow 0$ the full distribution becomes extremely peaked and, although the 


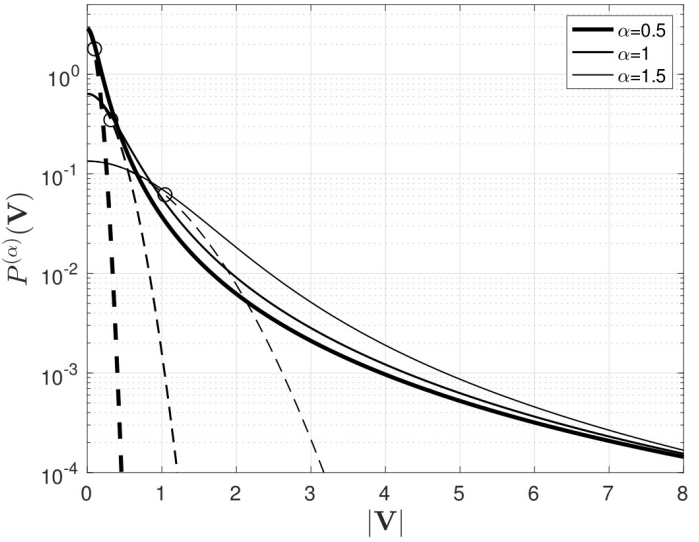

(a)

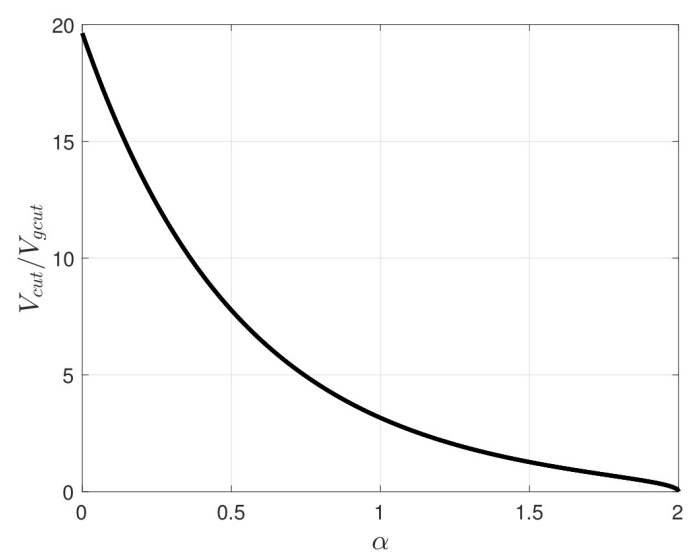

(c)



(b)

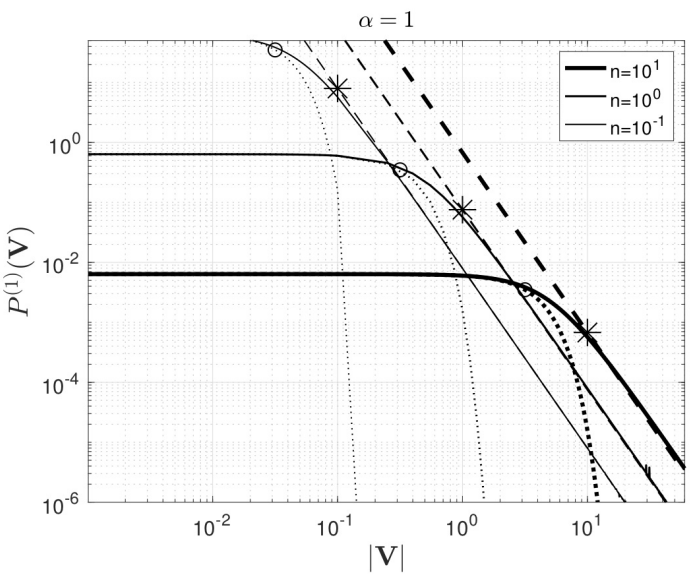

(d)

Figure 3. Comparison of the full distribution 49 with the approximation 55. Panel (a) shows the PDFs (continuous lines) in semilogarithmic scale for fixed density $n=1$ and circulation $\gamma=1$, for different values of locality degree $\alpha=0.5,1,1.5$, and the corresponding Gaussian functions (dashed lines) for the small velocity limit approximation. The circles represent the respective values for $V_{\text {gcut }}$. Panel (b) shows the previous distributions in logarithmic scale and the tail (dashed lines) approximations. The stars represent the respective values of $V_{\text {cut }}$. Panel (c) shows the ratio between $V_{\text {cut }}$ and $V_{\text {gcut }}$. Panel (d) shows the case $\alpha=1$, SQG, for three different values of the density $n=10^{1}, 10^{0}, 10^{-1}$ and $\gamma=1$. As before dotted lines represent the small velocity approximations, dashed lines the power law tail approximations, and the circles and stars the limits of validity for the approximations.

limits of validity for both approximations decrease, the ratio between them has a maximum. For increasing values of $\alpha$ the ratio decreases, reaching the value of zero for $\alpha \rightarrow 2$, showing thus that the distribution tends to become a Gaussian with diverging variance. This is a consequence of the fact that in $2 D$ turbulence the thermodynamic limit is not defined. The ratio $V_{\text {cut }} / V_{\text {gcut }} \sim 1$ when $\alpha \sim 1.62$. For this value of $\alpha$ it is thus possible to build the full distribution simply by pasting the two approximations together.

Finally, Figure $3 \mathrm{~d}$ shows the SQG distribution, $\alpha=1$, and its approximations for different values of the density. In this case $V_{\text {cut }} / V_{\text {gcut }} \sim 3.16$, so for the SQG case the value of $V_{\text {cut }}$ is always around three times the value of $V_{\text {gcut }}$. 


\subsection{Further analytical remarks}

\subsubsection{Non-neutral systems}

The distribution (55) has been derived by using assumption (i), i.e. assuming that the total system is neutral. If we want to take into account also a possible solid rotation for the system, we must consider that the average velocity increases linearly with the distance and the probability distribution gains a spatial dependency. At a point $\boldsymbol{r} \neq 0$ the distribution (55) must be modified by replacing the velocity $\boldsymbol{V}$ with the fluctuation

$$
\mathcal{V}=\boldsymbol{V}-\langle\boldsymbol{V}\rangle=\boldsymbol{V}-\frac{1}{2} n \gamma \boldsymbol{r}_{\perp}
$$

so that

$$
P^{(\alpha)}(\mathcal{V}) \sim \begin{cases}\frac{\left(c^{(\alpha)}\right)^{\alpha-3} \Gamma(4-\alpha)}{4 \pi} \exp \left(-\frac{1}{4\left(c^{(\alpha)}\right)^{3-\alpha}} \frac{\Gamma(6-2 \alpha)}{\Gamma(3-\alpha)} \mathcal{V}^{2}\right), & \mathcal{V}<\mathcal{V}_{\text {gcut }} \\ \frac{n(\gamma|\Psi(\alpha)|)^{\frac{2}{3-\alpha}}}{3-\alpha} \mathcal{V}^{-2 \frac{4-\alpha}{3-\alpha}}, & \mathcal{V}>\mathcal{V}_{\text {cut }}\end{cases}
$$

\subsubsection{Remarks on $\alpha \geq 2$}

In order to obtain the probability distribution, we have considered $n C^{(\alpha)}(\boldsymbol{\rho}) / N \ll 1$ in the limiting process (18). This limit can however be considered valid just for $\alpha \neq 2$. In the case in which $\alpha=2$, Figure 2 shows in fact that $\kappa^{(\alpha)}$ diverges and the special limit can not be used. We can thus conclude that, although we could expect the variance to diverge when the dynamics becomes less local, the shape of the probability distribution is not to be considered correct when $\alpha \rightarrow 2$.

For $\alpha>2, \kappa^{(\alpha)}$ has a discontinuous behaviour, and assumes both positive and negative values. However, when $\kappa^{(\alpha)}>0$, e.g. in the interval $\alpha \in(2.5,2.66]$, one can use exactly the same strategy for the computation of the probability, both for the core and the tails. The results here derived will thus hold also for some of the $\alpha$-models in the nonlocal dynamics.

\subsubsection{Equivalence between nonuniform configurations of point vortices}

In general for different values of $\alpha$ we can found different PDFs for the velocity field generated by the vortices. However, it is possible to connect the statistics of the velocity field of point vortices uniformly distributed for local $\alpha$-models in classical turbulence, with the statistics of the velocity field for point vortices non-uniformly distributed, that are relevant e.g. for quantum turbulence (Bradley and Anderson 2012, Reeves et al. 2013, 2014, Skaugen and Angheluta 2016a). For example, using the damped Gross-Pitaevskii equation with a stirring potential, it is possible to simulate a statistically steady state turbulent regime of a 2D trapped Bose-Einstein condensates, where vortices are emitted in clusters in the wake of the stirring obstacle (Skaugen and Angheluta 2016b). The statistic of the velocity field is an indicator of the vortex clustering and can be used to determine if the quantum turbulence exhibits an inverse energy cascade.

Consider an ensemble of $N$ identical point vortices on a disk of radius $R$ for an $\alpha$-model described by $\alpha_{1}=2$, and distributed following the probability density

$$
p_{(\beta)}(\boldsymbol{r})=\frac{n_{(\beta)}}{N}|\boldsymbol{r}|^{-\beta-1}, \quad \beta \in(-1,1),
$$

where $|\boldsymbol{r}|$ is the distance to the origin of the disk and

$$
n_{(\beta)}=\frac{N(1-\beta)}{2 \pi R^{1-\beta}}
$$

is the fractal density, that is, the cluster is self-similar with fractal dimension $1-\beta$. For $\beta=1 / 3$

the distribution of the clustered vortices is associated with an inverse cascade in $2 \mathrm{D}$ quantum 
turbulence. Following Skaugen and Angheluta (2016a), Chavanis (2009), it is possible to find the probability for the vortices fluctuation as

$$
P_{(\beta)}^{(2)}(\boldsymbol{V})=\frac{1}{(2 \pi)^{2}} \int_{0}^{2 \pi} \int_{0}^{\infty} \rho e^{-n_{(\beta)} C_{(\beta)}^{(2)}(\boldsymbol{\rho})} e^{i \rho V \cos \theta} d \rho d \theta
$$

where

$$
\begin{aligned}
C_{(\beta)}^{(2)}(\boldsymbol{\rho}) & =2 \pi\left(\frac{\gamma \rho}{2 \pi}\right)^{1-\beta} \int_{0}^{\infty}\left(1-J_{0}(x)\right) x^{\beta-2} d x \\
& =2 \pi \kappa_{(\beta)}^{(2)}\left(\frac{\gamma \rho}{2 \pi}\right)^{1-\beta}
\end{aligned}
$$

being $\kappa_{(\beta)}^{(2)}$ the number coming from the integral, that for the values of $\beta$ in the interval considered is positive.

These results are similar to the ones found using uniform distribution for the point vortices and different $\alpha$-models in the thermodynamic limit, that is $R, N \rightarrow \infty$ with constant density. The distribution of the velocity for $\alpha_{1}=2$ point vortices non-uniformly distributed with fractal dimension $1-\beta,(60)$, can be related to the distribution of the velocity for any $\alpha_{2} \in(0,2)$ point vortices distributed uniformly in space, for classical turbulence, by setting

$$
\beta=\frac{1-\alpha_{2}}{3-\alpha_{2}} \quad \forall \alpha_{2} \in(0,2) \text { and } \alpha_{1}=2,
$$

and rescaling the circulation of the $\alpha_{1}$-model as

$$
\gamma \rightarrow \gamma \frac{2 \pi\left|\Psi\left(\alpha_{2}\right)\right|}{\left(3-\alpha_{2}\right)^{\frac{3-\alpha_{2}}{2}}} .
$$

Clearly, when the solution of (31) is considered, we have also to substitute $n_{(\beta)}$ with $n$ of (12). Since we are considering local dynamics, i.e. $\alpha_{2} \in(0,2)$, then the only possible values for $\beta$ are in the interval $(-1,1 / 3)$. Note that $\beta=1 / 3$ can be considered only if $\alpha_{2}=0$, a non relevant case for classical turbulence.

The relations above show that the statistics of the velocity for point vortices with circulation $\gamma$ uniformly distributed in the SQG framework, $\alpha_{2}=1$, are the same of the ones for point vortices with $\alpha_{1}=2$, but distributed as

$$
p_{(0)}(\boldsymbol{r})=\frac{n_{(0)}}{N}|\boldsymbol{r}|^{-1}
$$

where

$$
n_{(0)}=\frac{N}{2 \pi R},
$$

and with a circulation scaled following (63), that is $\gamma / \pi|\Psi(1)|$.

It is clearly also possible to make a generalization relating the statistics of $\alpha_{2}$-model, characterized by uniform distribution of the vortices, with $\alpha_{1}$-model, $\alpha_{1} \neq 2$, that are distributed following a power law distribution using

$$
\beta=\frac{2 \alpha_{1}-\alpha_{2}-3}{3-\alpha_{2}} \quad \forall \alpha_{2} \text { and } \alpha_{1} \neq 2,
$$

and setting in the $\alpha_{1}$-model

$$
\gamma \rightarrow \frac{\gamma}{\Psi\left(\alpha_{1}\right)}\left(\frac{3-\alpha_{1}}{4 \pi^{2}}\right)^{3-\alpha_{2}} \quad \alpha_{2}=2 \text { and } \alpha_{1} \neq 2
$$


and

$$
\gamma \rightarrow \gamma\left(\frac{3-\alpha_{1}}{3-\alpha_{2}}\right)^{\frac{3-\alpha_{2}}{2}} \quad \alpha_{2} \neq 2 \text { and } \alpha_{1} \neq 2 .
$$

For this general group of transformations $\beta \in(-5,1 / 3)$. Since we are studying a cluster of vortices in the plane, we could expect a fractal dimension $(1-\beta) \in(0,2)$ and so, only the combination of $\alpha_{1}$ and $\alpha_{2}$ for which $\beta \in(-1,1 / 3)$ should be considered.

\section{Numerical simulations and finite size effects}

The analytical results illustrated in this work make use of the point vortex approximation, a strong simplification of the original problem. In order to test the reliability of the results when a fully turbulent field is considered without approximations, we perform numerical simulations of (1) and (2) for three different values of $\alpha$. In particular we consider $\alpha=1$, which correspond to SQG, and other two turbulent fields with degrees of locality that are smaller and greater than the SQG case, that is $\alpha=1.5$, and $\alpha=0.5$ respectively.

We will focus on the slope of the tails of the PDFs for the absolute value of the velocity field, which depends only on the degree of locality $\alpha$. In order to preserve the Hamiltonian structure of the system, the simulations are performed in a freely decaying setup.

The advection of the $\zeta$ field is performed using a semi-spectral method in a double periodic domain of length $L=2 \pi$. The horizontal resolution is set to $512 \times 512$ Fourier modes. We use a fourth order Runge-Kutta time stepping scheme with time step $\Delta t=0.005$. The simulations are performed up to $T=800$. The Jacobian is computed using the Arakawa (1966) discretization. To avoid accumulation of enstrophy at the small scales, we employ an exponential filter multiplying each Fourier mode by $\rho\left(2 K_{x} / N\right) \rho\left(2 K_{y} / N\right)$, where $\rho(x)=\exp \left(-a x^{m}\right)$ (Hou and Li 2007) and $K_{x}, K_{y}$ indicate, respectively, the zonal and meridional wavenumbers. Following Constantin et al. (2012), Ragone and Badin (2016) the coefficients of the filter are set to $a=36$ and $m=19$. This choice of the parameters ensures that approximately two-third of the low wavenumber modes remains unchanged.

The initial condition is set by a random $\zeta$ field obtained by inversion of the stream function

$$
\hat{\psi}(\mathbf{k}, 0) \propto \frac{k^{m / 4-1}}{\left(k+k_{0}\right)^{m / 2}},
$$

with $m=25$ and $k_{0}=6$ (Hakim et al. 2002, Ragone and Badin 2016) and where the hat indicates the Fourier transform of the quantity specified in physical space. The initial conditions for the three different simulations, corresponding to $\alpha=(1.5,1,0.5)$, have been normalized so that the quantity $E(\mathbf{k})=1 / 2 k^{2}|\hat{\psi}(\mathbf{k}, 0)|$, that in the SQG case represents the surface kinetic energy, is $E(\mathbf{k})=4 \pi^{2}(3,3,80)$ respectively. The higher value for the case of $\alpha=0.5$ is given by the fact that in this case the PDF of the velocity tends to be more peaked and the determination of the tail can thus be problematic. A more energetic field ensures thus a larger number of points in the tails of the distribution.

Each simulation reaches a quasi-steady state for which the two conserved quantities of the model, that is the generalized energy $E_{g}$ and the generalized enstrophy $Z_{g}$, defined as

$$
E_{g}=-\overline{\psi \zeta}, \quad Z_{g}=\frac{1}{2} \overline{\zeta^{2}}
$$

where the overbars indicate domain averages, are approximately constant. Once this quasisteady state is achieved, we compute $P(|\mathbf{V}|)$ for several eddy turnover times, defined for 
different values of the degree of locality $\alpha$ as

$$
T_{e d d y}=\frac{(2 \pi)^{2-\alpha}}{\zeta_{r m s}}
$$

The PDFs are then averaged in time between $T=400$ and $T=800$. In the simulations here performed one has $T_{e d d y} \sim 6.12$ for $\alpha=1.5$ and $T_{\text {eddy }} \sim 36.5$ for $\alpha=1$ and $\alpha=0.5$. The time average for the PDFs correspond thus averages over 65 eddy turnover times for $\alpha=1.5$ and 11 eddy turnover times for $\alpha=1$ and $\alpha=0.5$.

In the point vortex approximation, the velocity field decrease with power law (10), which has a maximum at the location of the vortex. Considering vortices with finite size, the velocity field grows from the center of the vortices, reaching a maximum at the vortex boundary, and decreases outside. To recover a situation similar to the one obtained using the point vortex approximation, we need to use a mask that allow us to consider the velocity field in between the vortices, excluding their interiors. This is approximately achieved considering region of the velocity field for which $\zeta \in[-\sigma(\zeta), \sigma(\zeta)]$, where $\sigma(\zeta)$ is the standard deviation of the $\zeta$ field. For the case $\alpha=0.5$ the strong forward cascade of $\zeta$ variance produces a large number of small vortices. In this case we thus use the interval $\zeta \in[-\sigma(\zeta) / 2, \sigma(\zeta) / 2]$ to avoid their inclusion in the region used for the computation of the PDFs.

\section{1. $\quad \boldsymbol{S Q G}(\alpha=1)$}

Figure 4 shows the SQG case, which corresponds to $\alpha=1$. The $\zeta$ field (Figure 4a) shows several well defined vortices as well as a number of secondary, smaller vortices in between them. Panels (b) and (c) show that the mask is able to discriminate between the regions inside and outside the vortices. The velocity field shows that inside the vortices the velocity increases from the center to the boundaries and decreases quickly outside of them (Figure 4 c).

For the SQG case, (37) yields

$$
P^{(1)}(|\boldsymbol{V}|=V) \sim \frac{2 \pi n \gamma|\Psi(1)|}{2} V^{-2} .
$$

The averaged PDF (Figure $4 \mathrm{~d}$ ) shows indeed tails with slope -2 , which is in agreement with the value predicted by the analytical point vortex model.

\section{2. $\alpha=1.5$}

Figure 5 shows the results for the case $\alpha=1.5$. The $\zeta$ field (Figure 5 a) shows several well defined vortices with a smaller number of secondary vortices in between them than for SQG. Also in this case, panels (b) and (c) show that the mask is able to discriminate between the regions inside and outside the vortices. The comparison between Figures 5 c and 4 c shows that for $\alpha=1.5$ the vortices have broader borders, characterised by high velocities, than for SQG. For $\alpha=1.5$, the analytical results predict a slope for the tails of the PDF of $-7 / 3=-2 . \overline{3}$. The numerical results show that the tail of the averaged PDF for the absolute value of the velocity field follows almost exactly the power law found by the analytical result, with a fit for the tail region highlighted by the length of the dotted line of -2.4 (Figure $5 \mathrm{~d}$ ). Notice that

the core of the PDF exhibits a secondary peak caused by the finite size of the vortices and not predicted by the point vortex approximation.

\section{3. $\alpha=0.5$}

Finally, Figure 6 shows the results for $\alpha=0.5$. The $\zeta$ field (Figure 6 ) shows strong secondary instabilities which span big portion of the space in the domain. The numerically estimated 




(a) $\zeta$

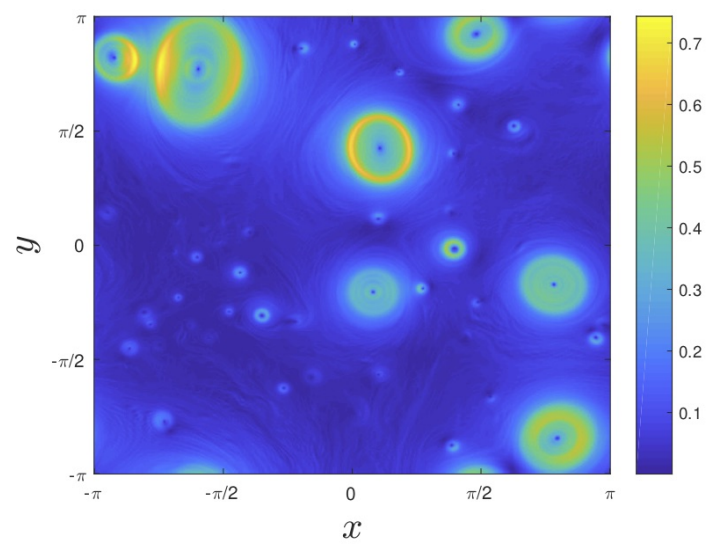

(c) $|\mathbf{V}|$

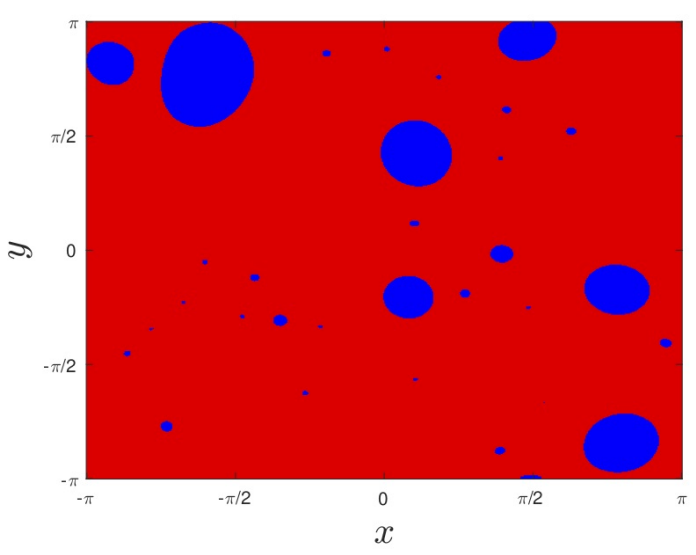

(b) Mask

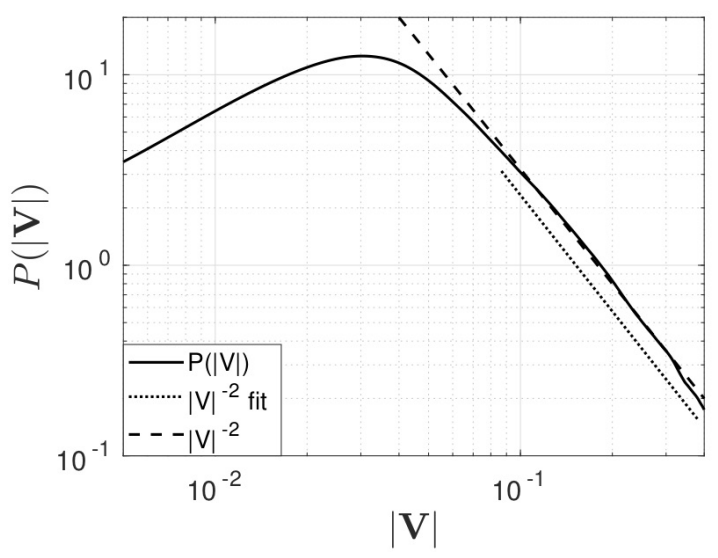

(d) $P(|\mathbf{V}|)$

Figure 4. Numerical simulation of 1 and 2 with $\alpha=1$. Panel (a) shows the last snapshot of the simulation for the $\zeta$ field. The range of variability is limited to $[-\sigma(\zeta), \sigma(\zeta)]$. Panel (b) shows the mask used for the computation of the PDF with respect to the previous panel. Panel (c) shows the map for the module of the velocity, $|\mathbf{V}|$, in the last snapshot of the simulation. Panel (d) shows the PDF (solid line) for the module of the velocity computed over several eddy turnover time (71), the power law approximation (36) (dashed line), and the fit of the tail of the PDF (dotted line).

slope of the tails for this case is of -1.9 , which is in good agreement with the value predicted by the theory of $-9 / 5=-1.8$.

Notice that the finite size effects of the vortices could be introduced in the analytical model by the inclusion of a finite radius $a$, so the maximum allowable velocity is $V=\gamma|\Psi(\alpha)| /(2 a)^{3-\alpha}$ obtained when the two vortices are at distance $\approx 2 a$ from each others. Infinite velocity and collapse of vortices are avoided. On the phenomena of collapse, see e.g. Aref (1979), Novikov and Sedov (1979), O'Neil (1987), Tavantzis and Ting (1988), Kimura (1990), Leoncini et al. (2000, 2001), Hernández-Garduno and Lacomba (2007), Sakajo (2008, 2012), Kudela (2014), Badin and Barry (2018).

\section{Conclusions}

In this work we have investigated the velocity statistics for turbulent flows, with the simplification of point vortices randomly distributed. The interactions of the point vortices are ruled by a family of $\alpha$-models in the local dynamics, which include the physically and mathematically 




(a) $\zeta$

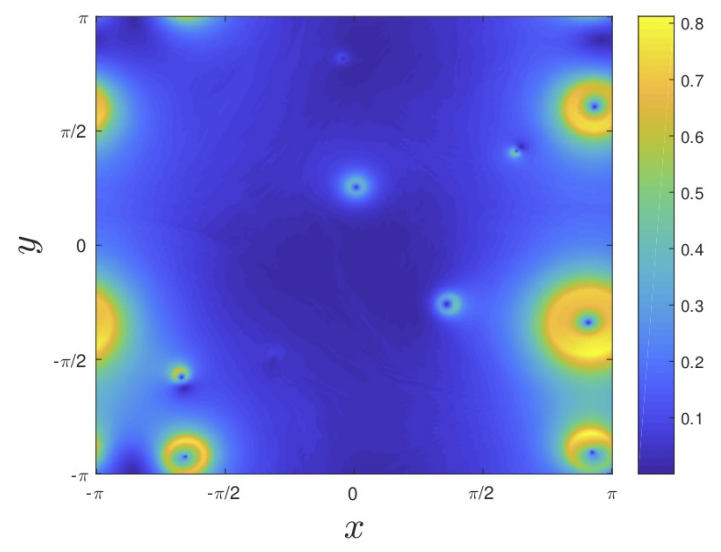

(c) $|\mathbf{V}|$

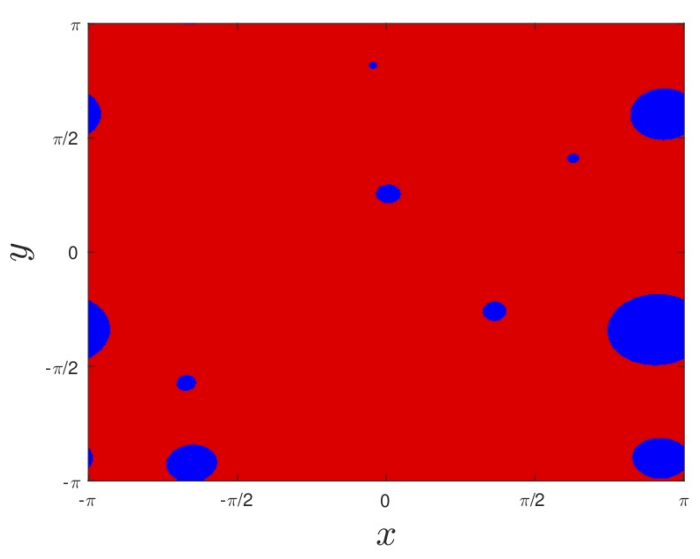

(b) Mask

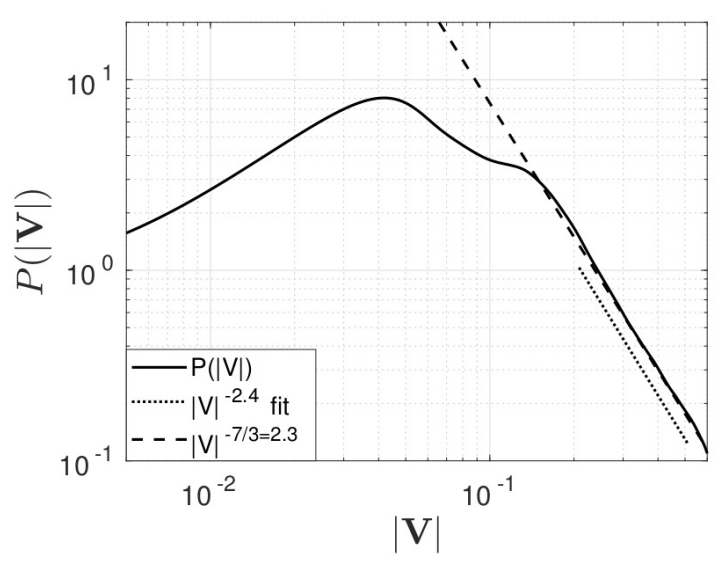

(d) $P(|\mathbf{V}|)$

Figure 5. As in Figure 4 but for $\alpha=1.5$.

interesting SQG model. The local dynamics differ from the case of $\alpha=2$ as we are able to consider the true thermodynamic limit, that is, if $N$ is the number of point vortices into a disc of radius $R$, we can consider the limits $N \rightarrow \infty, R \rightarrow \infty$ keeping the density $n$ constant. This limit is not defined for $\alpha=2$. As a consequence of this fact, the distributions that we obtain are well defined and independent on the number of point vortices in the domain considered.

This result clearly shows that the case $\alpha=2$ must be considered as a singular limit for the dynamics. The passage from local to nonlocal dynamics corresponds to a change in the topology of the system, and the passage between the two regimes must not be considered as continuous.

Results show that he central region of the distribution is not a Gaussian curve, in contrast to the case of $2 D$ turbulence. However, in the small velocity limit we can use a Gaussian function to approximate the peak of the distribution. The tail of the distribution follows a power law. Both the variance of the Gaussian approximation and the slope of the tail depend parametrically on $\alpha$. It is interesting to remark that the tail of the distribution exhibits selfsimilarity in respect to the density parameter.

Turbulence in the atmosphere and in the ocean is often characterized by means of wavenumber turbulent spectra. These can be used to infer for example if dynamics are either local or nonlocal. Very often the slopes of the inertial range in geophysical fluids are however difficult to obtain from observations or they can be ambiguous in their interpretation. The results 


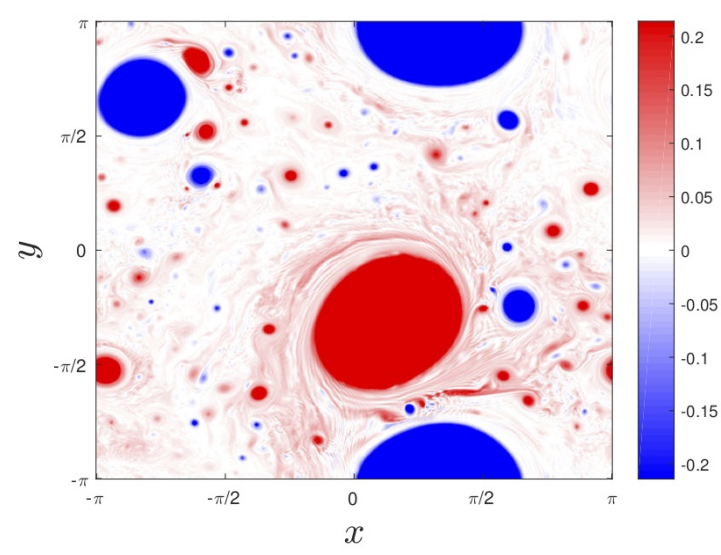

(a) $\zeta$

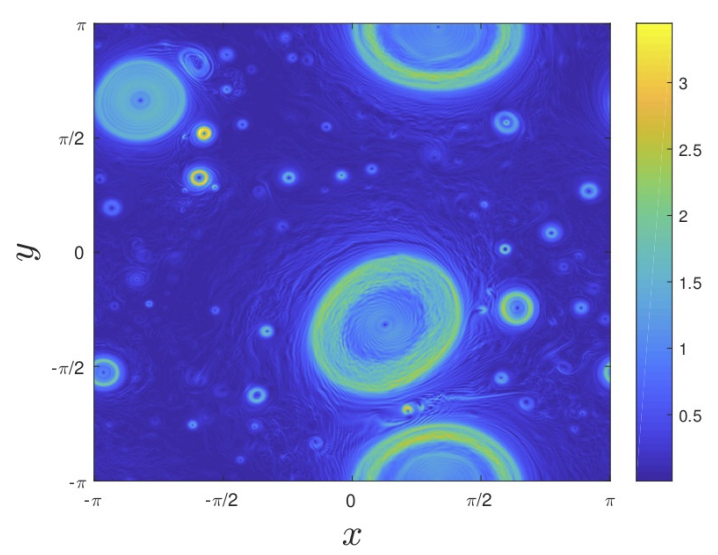

(c) $|\mathbf{V}|$

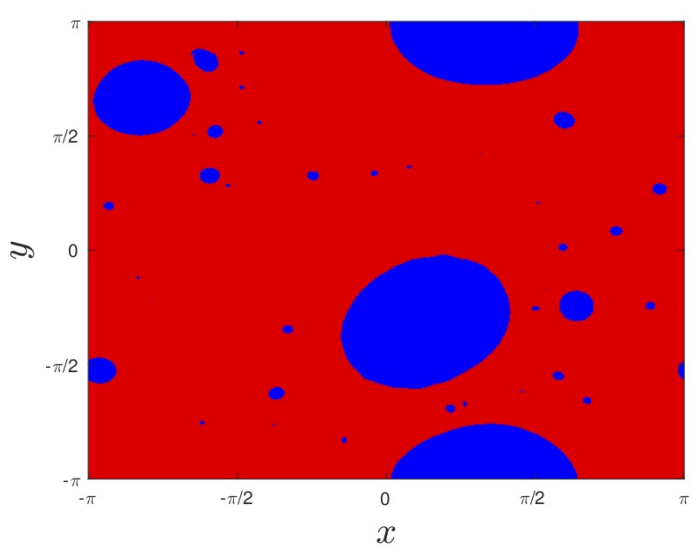

(b) Mask

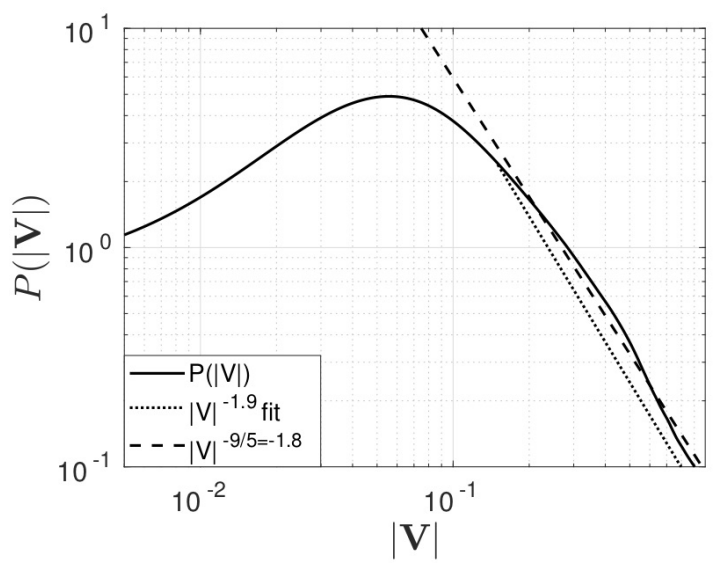

(d) $P(|\mathbf{V}|)$

Figure 6. As in Figure 5 but for $\alpha=0.5$. In this case the range of variability of $\zeta$, and the corresponding mask, are in the interval $[-\sigma(\zeta) / 2, \sigma(\zeta) / 2]$

obtained in the current study suggest however that the local nature of the turbulent fluctuations might be deduced from the shape of the tails of the PDFs. The velocity's PDFs for the barotropic turbulence in the ocean has been studied in Bracco et al. (2000b a)

Finally, we have also observed a connection between the statistics of the velocity field of point vortices uniformly distributed for local $\alpha$-models in classical turbulence, with the statistics of the velocity field for point vortices non-uniformly distributed, that are relevant for quantum turbulence. In particular the statistics of the velocity for point vortices uniformly distributed in the SQG framework, are the same of the ones for point vortices following the 2D quantum turbulence and distributed following a probability density $p(\boldsymbol{r}) \sim \boldsymbol{r}^{-1}$.

It should be noted that the $\alpha$-models here analysed can be used to represent also higher order balanced models of geophysical flows, such as the surface semi-geostrophic (SSG) model (Badin 2013, Ragone and Badin 2016). The velocity fluctuations for these higher order models are still unexplored and might give important insights on the physics of these systems.

\section{Acknowledgements}

GB would like to thank A.M. Barry for discussions on point-vortex dynamics in the $\alpha$-models. This study was partially funded by the DFG research grant BA 5068/9-1. GB was partially funded also by the DFG research grants TRR181, BA 5068/8-1 and 1740 . 


\section{Appendix A: Derivation of (37)}

Following Chavanis and Sire (2000), Chavanis (2009) and setting $t=\cos \theta$ and $z=\rho V$, (31) can be written as

$$
P^{(\alpha)}(\boldsymbol{V})=\frac{1}{2 \pi^{2} V^{2}} \int_{-1}^{1} \frac{d t}{\sqrt{1-t^{2}}} \int_{0}^{\infty} z e^{i z t} e^{-n C^{(\alpha)}(z / V)} d z,
$$

where we have exploited the symmetry of the cosine function to restrict the polar integration between 0 and $\pi$. These substitutions allow to study the velocity PDF when $V \rightarrow \infty$ as a power series of $z / V$. In order to perform the expansion and integrate term by term, it is necessary to consider the variables as complex, and change the path of the integration to ensure the analyticity of the inner integrand. The path of $t$ can be deformed to the unit semicircle $\tau$ in the positive imaginary half plane. In this way $\arg t$ can vary continuously between 0 and $\pi$. Rotating the integration path for $z$ of an angle $\omega(t)$ that depends on $\arg t$, we can ensure the convergence for $e^{i z t}$. The real part of $i z t$ must be negative, that is $\arg (i z t)=\pi / 2+\omega(t)+\arg t$ must be chosen so that it is kept between $\pi / 2$ and $3 \pi / 2$. In the same way the real part of $C^{(\alpha)}(z / V) \propto z^{2 /(3-\alpha)}$ should be positive, so that also the second exponential goes to zero for large $z$. This means that $\arg \left(z^{2 /(3-\alpha)}\right)=(2 /(3-\alpha)) \omega(t)$ should be kept in the interval $-\pi / 2$ and $\pi / 2$. In order to satisfy these constraints a possible choice for $\omega$ is

$$
\omega(t)=\frac{1}{8}\left(\frac{\pi}{2}-\arg t\right) .
$$

In this way $\omega(t)$ varies between $-\pi / 16$ and $\pi / 16$ and then $(2 /(3-\alpha)) \omega(t)$ respects the right condition explained above. After these necessary prescriptions we can expand the integrand

$$
\begin{aligned}
P^{(\alpha)}(\boldsymbol{V}) & =\frac{1}{2 \pi^{2} V^{2}} \sum_{m=0}^{\infty} \frac{1}{m !}\left(-\frac{2 \pi \kappa^{(\alpha)} n}{3-\alpha}\right)^{m}(\gamma|\Psi(\alpha)|)^{\frac{2 m}{3-\alpha}} \\
& \times\left(\frac{1}{V}\right)^{\frac{2 m}{3-\alpha}} \int_{\tau} \frac{d t}{\sqrt{1-t^{2}}} \int_{\omega(t)} z e^{i z t} z^{\frac{2 m}{3-\alpha}} d z .
\end{aligned}
$$

These integrals are convergent along any line where the real part of $i z t$ is negative, so we can rotate again the axis for $z$ considering the rotation $\omega(t)=\pi-\arg t$. Along this new integration path we also change the variable, $y=-i z t$, obtaining

$$
\begin{aligned}
P^{(\alpha)}(\boldsymbol{V}) & =-\frac{1}{2 \pi^{2} V^{2}} \sum_{m=0}^{\infty} \frac{1}{m !}\left(\frac{-2 \pi \kappa^{(\alpha) n}}{3-\alpha}\right)^{m}(\gamma|\Psi(\alpha)|)^{\frac{2 m}{3-\alpha}} \\
& \times\left(\frac{i}{V}\right)^{\frac{2 m}{3-\alpha}} \int_{\tau} \frac{d t}{\sqrt{1-t^{2}} t^{2+\frac{2 m}{3-\alpha}}} \int_{0}^{\infty} e^{-y} y^{\frac{2 m}{3-\alpha}+1} d y .
\end{aligned}
$$

Since

$$
\int_{\tau} \frac{d t}{\sqrt{1-t^{2}} t^{l}}=-\frac{i}{2}\left(1-e^{i \pi l}\right) B\left(\frac{m}{2}, \frac{1}{2}\right)
$$

the first term of the expansion, $m=0$, vanishes (see, e.g. Skaugen and Angheluta (2016a)). For $m=1$ we have

$$
\int_{\tau} \frac{d t}{\sqrt{1-t^{2}} t^{2 \frac{4-\alpha}{3-\alpha}}}=-\frac{i}{2}\left(1-e^{-i \pi 2 \frac{4-\alpha}{3-\alpha}}\right) B\left(\frac{4-\alpha}{3-\alpha}, \frac{1}{2}\right)
$$

while the integral on $y$ for the $m=1$ is the $\Gamma$ function

$$
\int_{0}^{\infty} e^{-y} y^{\frac{5-\alpha}{3-\alpha}} d y=\Gamma\left(2 \frac{4-\alpha}{3-\alpha}\right)
$$


Combining all the results together (37) is obtained at the leading order.

\section{References}

Arakawa, A., Computational design for long-term numerical integration of the equations of fluid motion: Twodimensional incompressible flow. Part I. J. Comp. Phys., 1966, 1, 119-143.

Aref, H., Motion of three vortices. Phys. Fluids, 1979, 22, 393-400.

Aref, H., Point vortex dynamics: a classical mathematics playground. J. Math. Phys., 2007, 48, 065401.

Badin, G., Surface semi-geostrophic dynamics in the ocean. Geophys. Astrophys. Fluid Dyn., 2013, 107, 526540.

Badin, G., On the role of non-uniform stratification and short-wave instabilities in three-layer quasi-geostrophic turbulence. Phys. Fluids, 2014, 26, 096603.

Badin, G. and Barry, A.M., Collapse of generalized Euler and surface quasigeostrophic point vortices. Phys. Rev. E, 2018, 98, 023110.

Badin, G. and Crisciani, F., Variational Formulation of Fluid and Geophysical Fluid Dynamics: Mechanics, Symmetries and Conservation Laws, 2018 (Springer).

Badin, G. and Poulin, F., Asymptotic scale-dependent stability of surface quasi-geostrophic vortices: semianalytic results. Geophys. Astro. Fluid Dyn., 2018 Doi:10.1080/03091929.2018.1453930.

Badin, G., Tandon, A. and Mahadevan, A., Lateral mixing in the pycnocline by baroclinic mixed layer eddies. J. Phys. Oceanogr., 2011, 41, 2080-2101.

Bembenek, E., Poulin, F. and Waite, M., Realizing surface-driven flows in the primitive equations. J. Phys. Oceanogr., 2015, 45, 1376-1392.

Blender, R. and Badin, G., Hydrodynamic Nambu mechanics derived by geometric constraints. J. Phys. A: Math. Theor., 2015, 48, 105501.

Blumen, W., Uniform potential vorticity flow: Part I. Theory of wave interactions and two-dimensional turbulence. J. Atmos. Sci., 1978, 35, 774-783.

Bracco, A., LaCasce, J.H. and Provenzale, A., Velocity Probability Density Functions for Oceanic Floats. J. Phys. Oceanogr., 2000a, 30, 461-474.

Bracco, A., LaCasce, J., Pasquero, C. and Provenzale, A., The velocity distribution of barotropic turbulence. Phys. Fluids, 2000b, 12, 2478-2488.

Bradley, A.S. and Anderson, B.P., Energy Spectra of Vortex Distributions in Two-Dimensional Quantum Turbulence. Phys. Rev. X, 2012, 2, 041001.

Bühler, O., Statistical mechanics of strong and weak point vortices in a cylinder. Phys. Fluids, 2002, 14, 2139-2149.

Burgess, B., Scott, R. and Shepherd, T., Kraichnan-Leith-Batchelor similarity theory and two-dimensional inverse cascades. J. Fluid Mech., 2015, 767, 467-496.

Burgess, B. and Shepherd, T., Spectral non-locality, absolute equilibria and Kraichnan-Leith-Batchelor phenomenology in two-dimensional turbulent energy cascades. J. Fluid Mech., 2013, 725, 332-371.

Capet, X., Klein, P., Hua, B., Lapeyre, G. and McWilliams, J., Surface kinetic energy transfer in surface quasi-geostrophic flows. J. Fluid Mech., 2008, 604, 165-174.

Carton, X., Instability of surface quasigeostrophic vortices. J. Atmos. Sci., 2009, 66, 1051-1062.

Carton, X., Ciani, D., Verron, J., Reinaud, J. and Sokolovskiy, M., Vortex merger in surface quasi-geostrophy. Geophys. Astro. Fluid Dyn., 2016, 110, 1-22.

Chandrasekhar, S., Statistical Theory of Stellar Encounters. Astrophys. J., 1941, 94, 511.

Chandrasekhar, S. and von Neumann, J., The Statistics of the Gravitational Field Arising from a Random Distribution of Stars, I. The Speed of Fluctuations. Astrophys. J., 1942, 95, 489-531.

Chandrasekhar, S. and von Neumann, J., The Statistics of the Gravitational Field Arising from a Random Distribution of Stars. II, The Speed of Fluctuations; Dynamical Friction; Spatial Co-Relations. Astrophys. J., 1943, 97, 1-27.

Chapman, D., Ideal vortex motion in two dimensions: Symmetries and conservation laws. J. Math. Phys., 1978, 19, 1988-1992.

Charney, J., Geostrophic turbulence. J. Atmos. Sci., 1971, 28, 1087-1095.

Chavanis, P.H., From Jupiter's Great Red Spot to the Structure of Galaxies: Statistical Mechanics of TwoDimensional Vortices and Stellar Systems. Ann. New York Acad. Sci., 1998, 867, 120-140.

Chavanis, P.H., Statistics of the gravitational force in various dimensions of space: from Gaussian to Lévy laws. Eur. Phys. J. B, 2009, 70, 413-433.

Chavanis, P.H. and Sire, C., Statistics of velocity fluctuations arising from a random distribution of point vortices: The speed of fluctuations and the diffusion coefficient. Phys. Rev. E, 2000, 62, 490-506.

Constantin, P., Lai, M.C., Sharma, R., Tseng, Y.H. and Wu, J., New numerical results for the surface quasigeostrophic equation. J. Sci. Comp., 2012, 50, 1-28.

Constantin, P., Majda, A. and Tabak, E., Formation of strong fronts in the 2-D quasigeostrophic thermal active scalar. Nonlinearity, 1994a, 7, 1495-1533. 
Constantin, P., Majda, A. and Tabak, E., Singular front formation in a model for quasigeostrophic flow. Phys. Fluids, 1994b, 6, 9-11.

Constantin, P., Nie, Q. and Schörghofer, N., Nonsingular surface quasi-geostrophic flow. Phys. Lett. A, 1998, 241, 168-172.

Constantin, P. and Wu, J., Behavior of solutions of 2D quasi-geostrophic equations. SIAM J. Math. Anal., 1999, 30, 937-948.

Córdoba, A. and Córdoba, D., A maximum principle applied to quasi-geostrophic equations. Comm. Math. Phys., 2004, 249, 511-528.

Córdoba, D. and Fefferman, C., Growth of solutions for QG and 2D Euler equations. J. Am. Math. Soc., 2002a, 15, 665-670.

Córdoba, D. and Fefferman, C., Scalars convected by a two-dimensional incompressible flow. Comm. Pure Appl. Math., 2002b, 55, 255-260.

Córdoba, D., Fontelos, M., Mancho, A. and Rodrigo, J., Evidence of singularities for a family of contour dynamics equations. Proc. Natl. Acad. Sci., 2005, 102, 5949-5952.

Deng, J., Hou, T., Li, R. and Yu, X., Level set dynamics and the non-blowup of the 2D quasi-geostrophic equation. Methods Appl. Anal., 2006, 13, 157-180.

Dong, H. and Li, D., Finite time singularities for a class of generalized surface quasi-geostrophic equations. Proc. Am. Math. Soc., 2008, 136, 2555-2563.

Dritschel, D., An exact steadily rotating surface quasi-geostrophic elliptical vortex. Geophys. Astro. Fluid Dyn., 2011, 105, 368-376.

Esler, J., Equilibrium energy spectrum of point vortex motion with remarks on ensemble choice and ergodicity. Phys. Rev. Fluids, 2017, 2, 014703.

Foussard, A., Berti, S., Perrot, X. and Lapeyre, G., Relative dispersion in generalized two-dimensional turbulence. J. Fluid Mech., 2017, 821, 358-383.

Hakim, G.J., Snyder, C. and Muraki, D.J., A New Surface Model for Cyclone-Anticyclone Asymmetry. J. Atmos. Sci., 2002, 59, 2405-2420.

Harvey, B. and Ambaum, M., Perturbed Rankine vortices in surface quasi-geostrophic dynamics. Geophys. Astro. Fluid Dyn., 2011, 105, 377-391.

Harvey, B., Ambaum, M. and Carton, X., Instability of shielded surface temperature vortices. J. Atmos. Sci., 2011, 68, 964-971.

Held, I.M., Pierrehumbert, R.T., Garner, S.T. and Swanson, K.L., Surface quasi-geostrophic dynamics. J. Fluid Mech., 1995, 282, 1-20.

Helmholtz, H., Über Integrale der hydrodynamischen Gleichungen, welche den Wirbelbewegungen entsprechen. J. Reine Angew. Math., 1858, 55, 25-55.

Hernández-Garduno, A. and Lacomba, E., Collisions and regularization for the 3-vortex problem. J. Math. Fluid Mech., 2007, 9, 75-86.

Hou, T.Y. and Li, R., Computing nearly singular solutions using pseudo-spectral methods. J. Comp. Phys., 2007, 226, $379-397$.

Iwayama, T. and Watanabe, T., Green's function for a generalized two-dimensional fluid. Phys. Rev. E, 2010, 82, 036307.

Jimnez, J., Algebraic probability density tails in decaying isotropic two-dimensional turbulence. J. Fluid Mech., 1996, 313, 223-240.

Ju, N., Geometric constrains for global regularity of 2D quasi-geostrophic flows. J. Diff. Eq., 2006, 226, 54-79.

Kimura, Y., Parametric motion of complex-time singularity toward real collapse. Physica D, 1990, 46, 439-448.

Kraichnan, R. and Montgomery, D., Two-dimensional turbulence. Rep. Prog. Phys., 1980, 43, 547.

Kudela, H., Collapse of n-point vortices in self-similar motion. Fluid Dyn. Res., 2014, 46, 031414.

Lapeyre, G., Surface Quasi-Geostrophy. Fluids, 2017, 2, 7.

Leoncini, X., Kuznetsov, L. and Zaslavsky, G., Motion of three vortices near collapse. Phys. Fluids, 2000, 12, 1911-1927.

Leoncini, X., Kuznetsov, L. and Zaslavsky, G., Chaotic advection near a three-vortex collapse. Phys. Rev. E, 2001, 63, 036224.

Li, D., Existence theorems for the 2D quasi-geostrophic equation with plane wave initial conditions. Nonlinearity, 2009, 22, 1639.

Lim, C. and Majda, A., Point vortex dynamics for coupled surface/interior QG and propagating heton clusters in models for ocean convection. Geophys. Astro. Fluid Dyn., 2001, 94, 177-220.

Majda, A. and Tabak, E., A two-dimensional model for quasigeostrophic flow: comparison with the twodimensional Euler flow. Physica D, 1996, 98, 515-522.

Marchand, F., Existence and Regularity of Weak Solutions to the Quasi-Geostrophic Equations in the Spaces $L^{p}$ or $\dot{H}^{-1 / 2}$. Comm. Math. Phys., 2008a, 277, 45-67.

Marchand, F., Weak-strong uniqueness criteria for the critical quasi-geostrophic equation. Physica D, 2008b, 237, 1346-1351.

Marchioro, C. and Pulvirenti, M., Mathematical theory of incompressible nonviscous fluids, Vol. 96, 2012 (Springer Science \& Business Media).

McWilliams, J.C., Submesoscale currents in the ocean. Proc. Roy. Soc. A, 2016, 472, 20160117.

Min, I.A., Mezic, I. and Leonard, A., Lévy stable distributions for velocity and velocity difference in systems of vortex elements. Phys. Fluids, 1996, 8, 1169-1180. 
Mukiibi, D., Badin, G. and Serra, N., Three-Dimensional Chaotic Advection by Mixed Layer Baroclinic Instabilities. J. Phys. Oceanogr., 2016, 46, 1509-1529.

Newton, P., The N-vortex problem: analytical techniques, Vol. 145, 2013 (Springer Science \& Business Media).

Novikov, E. and Sedov, Y., Vortex collapse. Zh. Eksp. Teor. Fiz., 1979, 77, 588-597.

Ohkitani, K. and Yamada, M., Inviscid and inviscid-limit behavior of a surface quasigeostrophic flow. Phys. Fluids, 1997, 9, 876-882.

Ohkitani, K., Asymptotics and numerics of a family of two-dimensional generalized surface quasi-geostrophic equations. Phys. Fluids, 2012, 24, 095101.

O'Neil, K., Stationary configurations of point vortices. Trans. Am. Math. Soc., 1987, 302, 383-425.

Onsager, L., Statistical hydrodynamics. Nuovo Cimento, 1949, 6, 279-287.

Pierrehumbert, R., Held, I. and Swanson, K., Spectra of local and nonlocal two-dimensional turbulence. Chaos Solit. Fract., 1994, 4, 1111-1116.

Ragone, F. and Badin, G., A study of surface semi-geostrophic turbulence: freely decaying dynamics. J. Fluid Mech., 2016, 792, 740-774.

Reeves, M.T., Billam, T.P., Anderson, B.P. and Bradley, A.S., Inverse Energy Cascade in Forced TwoDimensional Quantum Turbulence. Phys. Rev. Lett., 2013, 110, 104501.

Reeves, M.T., Billam, T.P., Anderson, B.P. and Bradley, A.S., Signatures of coherent vortex structures in a disordered two-dimensional quantum fluid. Phys. Rev. A, 2014, 89, 053631.

Rodrigo, J., On the evolution of sharp fronts for the quasi-geostrophic equation. Comm. Pure Appl. Math., $2005, \mathbf{5 8}, 821-866$.

Rodrigo, J. and Fefferman, C., The vortex patch problem for the surface quasi-geostrophic equation. Proc. Natl. Acad. Sci., 2004, pp. 2684-2686.

Sakajo, T., Non-self-similar, partial, and robust collapse of four point vortices on a sphere. Phys. Rev. E, 2008, 78, 016312.

Sakajo, T., Instantaneous energy and enstrophy variations in Euler- $\alpha$ point vortices via triple collapse. J. Fluid Mech., 2012, 702, 188-214.

Schorghofer, N., Universality of probability distributions among two-dimensional turbulent flows. Phys. Rev. E, 2000, 61, 6568-6571.

Scott, R., A scenario for finite-time singularity in the quasigeostrophic model. J. Fluid Mech., 2011, 687, $492-502$.

Scott, R. and Dritschel, D., Numerical simulation of a self-similar cascade of filament instabilities in the surface quasigeostrophic system. Phys. Rev. Lett., 2014, 112, 144505.

Shcherbina, A.Y., Sundermeyer, M.A., Kunze, E., D'Asaro, E., Badin, G., Birch, D., Brunner-Suzuki, A.M.E.G., Callies, J., Cervantes, B.T.K., Claret, M., Concannon, B., Early, J., Ferrari, R., Goodman, L., Harcourt, R.R., Klymak, J.M., Lee, C.M., Lelong, M.P., Levine, M.D., Lien, R.C., Mahadevan, A., McWilliams, J.C., Molemaker, M.J., Mukherjee, S., Nash, J.D., Özgökmen, T., Pierce, S.D., Ramachandran, S., Samelson, R.M., Sanford, T.B., Shearman, R.K., Skyllingstad, E.D., Smith, K.S., Tandon, A., Taylor, J.R., Terray, E.A., Thomas, L.N. and Ledwell, J.R., The LatMix Summer Campaign: Submesoscale Stirring in the Upper Ocean. BAMS, 2015, 96, 1257-1279.

Skaugen, A. and Angheluta, L., Velocity statistics for nonuniform configurations of point vortices. Phys. Rev. E, 2016a, 93, 042137.

Skaugen, A. and Angheluta, L., Vortex clustering and universal scaling laws in two-dimensional quantum turbulence. Phys. Rev. E, 2016b, 93, 032106.

Smith, K.S., Boccaletti, G., Henning, C., Marinov, I., Tam, C., Held, I. and Vallis, G.K., Turbulent diffusion in the geostrophic inverse cascade. J. Fluid Mech., 2002, 469, 13-48.

Tavantzis, J. and Ting, L., The dynamics of three vortices revisited. Phys. Fluids, 1988, 31, 1392-1409.

Taylor, C. and Llewellyn Smith, S., Dynamics and transport properties of three surface quasigeostrophic point vortices. Chaos, 2016, 26, 113117.

Tran, C., Nonlinear transfer and spectral distribution of energy in $\alpha$ turbulence. Physica D, 2004, 191, $137-155$.

Tran, C., Dritschel, D. and Scott, R., Effective degrees of nonlinearity in a family of generalized models of two-dimensional turbulence. Phys. Rev. E, 2010, 81, 016301.

Tran, C. and Shepherd, T., Constraints on the spectral distribution of energy and enstrophy dissipation in forced two-dimensional turbulence. Physica D, 2002, 165, 199-212.

Tulloch, R. and Smith, K., A theory for the atmospheric energy spectrum: Depth-limited temperature anomalies at the tropopause. Proc. Natl. Acad. Sci. U.S.A., 2006, 103, 14690-14694.

Venaille, A., Dauxois, T. and Ruffo, S., Violent relaxation in two-dimensional flows with varying interaction range. Phys. Rev. E, 2015, 92, 011001.

Weiss, J.B., Provenzale, A. and McWilliams, J.C., Lagrangian dynamics in high-dimensional point-vortex systems. Phys. Fluids, 1998, 10, 1929-1941.

Wu, J., Solutions of the 2D quasi-geostrophic equation in Hölder spaces. Nonlinear Anal., 2005, 62, 579-594. 